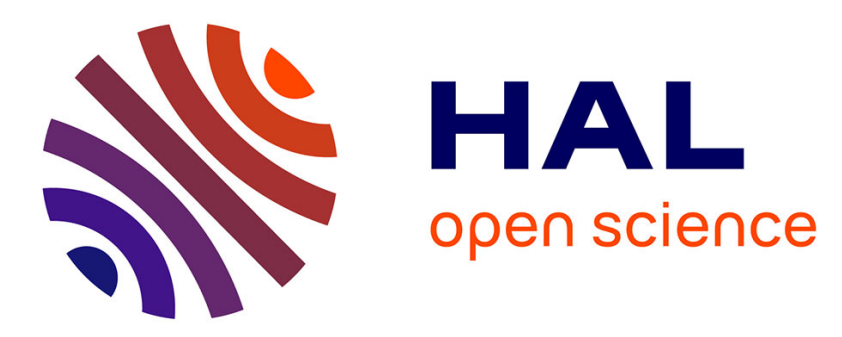

\title{
Ultra Low Power Consumption for Self-Oscillating Nanoelectromechanical Systems Constructed by Contacting Two Nanowires
}

Thomas Barois, Anthony Ayari, P. Vincent, S. Perisanu, P. Poncharal, S. T. Purcell

\section{To cite this version:}

Thomas Barois, Anthony Ayari, P. Vincent, S. Perisanu, P. Poncharal, et al.. Ultra Low Power Consumption for Self-Oscillating Nanoelectromechanical Systems Constructed by Contacting Two Nanowires. Nano Letters, 2013, 13 (4), pp.1451-1456. 10.1021/nl304352w · hal-01565117

\section{HAL Id: hal-01565117 https://hal.science/hal-01565117}

Submitted on 29 Sep 2017

HAL is a multi-disciplinary open access archive for the deposit and dissemination of scientific research documents, whether they are published or not. The documents may come from teaching and research institutions in France or abroad, or from public or private research centers.
L'archive ouverte pluridisciplinaire HAL, est destinée au dépôt et à la diffusion de documents scientifiques de niveau recherche, publiés ou non, émanant des établissements d'enseignement et de recherche français ou étrangers, des laboratoires publics ou privés. 


\title{
Ultra low power consumption for self-oscillating
} nanoelectromechanical systems constructed by contacting two nanowires

\author{
T. Barois, A. Ayari, ${ }^{*}$ P. Vincent, S. Perisanu, P. Poncharal, and S. T. Purcell \\ Laboratoire de Physique de la Matière Condensée et Nanostructures (UMR CNRS 5586), \\ Université Claude Bernard, 69622 Villeurbanne, France \\ E-mail: anthony.ayari@univ-lyon1.fr
}

\begin{abstract}
We report here the observation of a new self-oscillation mechanism in nanoelectromechanical systems (NEMS). A highly resistive nanowire was positioned to form a point-contact at a chosen vibration node of a silicon carbide nanowire resonator. Spontaneous and robust mechanical oscillations arise when a sufficient DC voltage is applied between the two nanowires. An original model predicting the threshold voltage is used to estimate the piezoresistivity of the point contact in agreement with the observations. The measured input power is in the $\mathrm{pW}$-range which is the lowest reported value for such systems. The simplicity of the contacting procedure and the low-power consumption open a new route for integrable and low-loss self-excited NEMS devices.
\end{abstract}

${ }^{*}$ To whom correspondence should be addressed 
Nanoelectromechanical systems (NEMS) have recently motivated much research in both fundamental physics and nanotechnology. For the former one can cite measuring mechanical ground state quantum oscillations ${ }^{1-3}$ and single electron resonance tuning ${ }^{4,5}$ and for the later, mass sensors with single atom resolution ${ }^{6}$ and radio receptors based on individual nanotube resonators. ${ }^{7-9}$ A well-established practical difficulty for NEMS applications is to be able to systematically find the resonance frequencies of such high Q-factor nanoscale resonators which vary somewhat arbitrarily due to fabrication tolerances and device drifts. A relevant option is to use NEMS with a self-oscillation ability for which only a DC energy supply is necessary for the spontaneous driving of the mechanical resonances. Furthermore, this approach is particularly interesting from the point of view of power saving when the input power is small and ultimately equal to the intrinsic mechanical losses of the resonators.

One class of self-oscillating NEMS has been developed using "external" positive feedback obtained from electrical amplifier loops ${ }^{10,11}$ and microwave ${ }^{12}$ or optical ${ }^{13-17}$ cavity fields. A major drawback is the large power consumption required to supply the external feedback while often only a single or a few nanoscale objects are spontaneously driven. A second promising class of self-oscillating NEMS uses "internal" positive feedback where the essential physical elements are all at the nanoscale, in close proximity to the resonator. The first such NEMS consisted of selfoscillating $\mathrm{SiC}$ nanowires in a field-emission configuration ${ }^{18}$. Carbon nanotubes (CNTs) can also be used as field-emitters for millimeter-sized mechanical self-oscillators ${ }^{19}$ or in a configuration where field-emitting nanotubes are the self-oscillating mechanical resonators. ${ }^{20}$ Self-oscillation during charge shuttling was first observed with molecules ${ }^{21}$ and then with nano-pillars ${ }^{22}$. Some specific transistors with either doped Si channels in depletion ${ }^{23}$ or CNT channels functioning in the Coulomb blockade regime ${ }^{4}$ have also shown mechanical self-oscillations. Other self-oscillation mechanisms are found with back-action mediated by thermal coupling ${ }^{24}$ or carrier excitation. ${ }^{25}$

In this article, we present a new internal feedback mechanism that leads to self-oscillations, demonstrated with an original device composed of two contacted nanowires. There are two particularly original aspects. Firstly, the device exploits a flexible contact at the nodes of the second or 
higher order vibration modes, a strategy that we have not seen in any previous NEMS. As a consequence the fundamental vibration mode is not dominant in the dynamics as is usual. Secondly, and more exceptionally, the contact makes use of nanoscale stiction effects, ${ }^{26}$ which are generally frequent and usually unwanted side effects during device fabrication or manipulation. Here the stiction between the nanowires forms a point-contact structure ${ }^{27}$ whose electromechanical properties are the key new element of this self-oscillation mechanism. The experimental observations are supported by an electromechanical model where the self-oscillation originates from the properties of the point-contact. The threshold voltage is used to determine a dynamical value for the piezoresistivity due to the mechanical oscillation of the point-contact. Besides the simplicity of the contacting technique, our observations show the existence of the self-oscillation regime for $\mathrm{nW}$ to $\mathrm{pW}$ of input power. This last value is promising as it is two orders of magnitude lower than previously reported values for self-oscillating NEMS devices. A table comparing the literature values of the efficiency of self-oscillation mechanisms is included. Our mechanism is found to have the highest value proving the interest for point-contact self-oscillating devices.

Two silicon carbide $(\mathrm{SiC})$ nanowires were mounted on electrochemically sharpened tungsten tips and introduced into an ultra high vacuum (UHV) chamber dedicated to nanomechanical measurements. The two tungsten support tips were fixed on Attocube piezo motors for relative XYZ positioning of the nanowires (see Fig. 1 (b)). The observation of the nanowire positions was made using a scanning electron microscope (SEM) integrated in the UHV chamber. The longer nanowire was the resonator $\left(L_{l}=200 \mu \mathrm{m}\right.$, diameter $\left.250 \mathrm{~nm}\right)$ while the shorter one served as a quasi immobile contact $\left(L_{s}=57 \mu \mathrm{m}\right.$, diameter $\left.350 \mathrm{~nm}\right)$. The two nanowires were approximately parallel to the SEM observation plane (XY) and the long resonator was displaced along the $\mathrm{Z}$ axis to place both nanowires simultaneously in the SEM focus plane. The XY displacements could be used to touch the apex of the long nanowire to either the tungsten support of the short nanowire or the short nanowire itself. The resistances are measured in this configuration with typical values $R_{l}=$ $1 \mathrm{G} \Omega$ for the long nanowire and $R_{s}=1 \mathrm{~T} \Omega$ for the short nanowire.

The position of the second mode's vibration node was determined using electrostatic driving 
before the sticking procedure (see supporting information, Fig. S1). The short nanowire was then contacted close to the node position (79 \% of the long nanowire length). The procedure for the contacting and the sticking of the nanowires is simple. The short nanowire was first moved close to the long nanowire. A DC voltage $(\sim 20 \mathrm{~V})$ was then applied between the two nanowires inducing an electrostatic attractive force between them. Once the nanowires touched, the adhesion forces at the contact were strong enough to maintain a rigid structure even when the bias voltage was reduced to zero. When an increasing DC voltage was applied between the two nanowires, the structure jumped spontaneously into mechanical self-oscillations at a specific threshold value $V_{t h}$ (figure 1 (a)). Furthermore, when the DC voltage applied to the oscillating system was swept downward, the oscillations disappeared abruptly at a lower extinction voltage, $V_{e}<V_{t h} . V_{t h}$ and $V_{e}$ delimit a rather large hysteresis cycle. Note that no AC driving was applied. A particularity of the oscillations was that the long nanowire underwent large amplitude vibrations while the movement of the short one was usually indiscernible. The DC current was measured with a series ammeter with pA resolution. During a voltage sweep, the appearance (disappearance) of the oscillations was concomitant with a sudden increase (decrease) in the current (figure 1 (c)). This means that the average conductivity of the structure increased when it oscillated.

The spontaneous oscillation frequency is measured by a time-resolved position detection technique in which the SEM electron beam is focalized on the long resonator near its base. ${ }^{28}$ During spontaneous oscillations the long resonator moves periodically in and out of the focused electron beam, thus creating a periodic secondary electron current that is measured by a scintillator. As the resonator intercepts the beam twice during one oscillation cycle, the frequency measured from the current signal is twice the oscillation frequency. During spontaneous oscillation, the number of cycles as a function of time is extracted from the acquired signal (see supporting information, Fig. S3). The number of cycles increased almost linearly with time (see the main plot in Fig. 2) giving a self-oscillation frequency of $36436 \pm 5$ cycles per second (i.e. Hz), very close to the eigenfrequency for the second mode of the free resonator $(38 \mathrm{kHz})$.

Several experimental evidence of the self-oscillation are to be noticed. Firstly, the existence of a 
DC threshold voltage suggests that the driving power of the vibration comes from the DC electrical source. Secondly, the stability of the frequency shown in Fig. 2 is typical of self-oscillators. As mentioned above, the number of cycle $\Pi(t)$ increases almost linearly with time $(\Pi(t) \sim<f>t)$. The fine evolution of the number of cycles is obtained when the average linear dependence is removed and thus defining the phase difference (cycles) $\Delta \Pi(t)=\Pi(t)-\langle f\rangle t$ where $\langle f\rangle$ is the average frequency. This phase difference $\Delta \Pi(t)$ fluctuates somewhat (see inserted plot in Fig. 2), meaning that the relative frequency $d \Delta \Pi(t) / d t$ is drifting on a timescale larger than $1 \mathrm{~ms}$. Finally the frequency increases with applied voltage (see supporting information, Fig. S4) meaning that this device acts as voltage-controlled oscillator (VCO).

A possible cause of the self-oscillations could have been the interaction of the resonator with the SEM column electron beam. ${ }^{29}$ To examine this possibility the IV characteristics were measured both with and without SEM imaging (see supporting information, Fig. S2). The same self-oscillations were observed in both cases meaning that the electron beam is not the physical source. As well, the DC threshold voltage was quite reproducible meaning that the beam had no or little influence on the resonator movement.

We propose that the self-oscillation regime originates from the internal environment of the contacted nanowires under a DC bias voltage where the nanoscale electromechanical properties of the point-contact play a crucial role. A simplified model is proposed for the description of the selfoscillation mechanism where only one mechanical degree of freedom is considered. The contact is assumed to be precisely at the vibration node which means that only the long resonator is likely to oscillate. Thus, the equation of motion for the vibration mode is:

$$
m \ddot{x}+\Gamma \dot{x}+k x=\mathscr{F}_{e}\left(V_{D C}, x, \dot{x}\right)
$$

where $x$ is the amplitude at the apex of the long resonator, $m$ is the effective mass of the vibration mode, $\Gamma$ the intrinsic damping, $k$ the mechanical equivalent spring constant and $\mathscr{F}_{e}\left(V_{D C}, x, \dot{x}\right)$ the electrostatic forcing. This forcing term comes from the electrostatic interaction between the two 
nanowires as the DC voltage introduces electrical charges on each nanowire. The dependencies in $x$ and $\dot{x}$ in $\mathscr{F}_{e}$ comes from the fact that the vibration of the long nanowire affects the values for the contact resistance and the coupling capacitance of the two nanowires thus affecting the charge distribution. Using a Taylor expansion for $\mathscr{F}_{e}\left(V_{D C}, x, \dot{x}\right)$, the equation of motion (Eq. 1) can be written as

$$
m \ddot{x}+\Gamma_{e} \dot{x}+k_{e} x=0
$$

where the effective spring coefficient $k_{e}=k+\partial \mathscr{F}_{e} / \partial x$ and the effective damping coefficient $\Gamma_{e}=\Gamma+\partial \mathscr{F}_{e} / \partial \dot{x}$ are introduced because of the electromechanical coupling. $\mathscr{F}_{e}$ is given in the supporting information as a function of the physical parameters of the nanowires. The selfoscillation threshold voltage $V_{t h}$ is obtained when the effective damping $\Gamma_{e}$ becomes zero:

$$
\Gamma_{e}=\Gamma+\frac{R_{t} C^{\prime} / 8+R^{\prime} /\left(2 R_{t}\right) \tau}{1+\omega_{0}^{2} \tau^{2}} C^{\prime} V_{t h}^{2}=0
$$

where $R_{t}$ is the total resistance of the contacted nanowires, $C$ the capacitance between them and $\tau=R_{t} C$ (see supporting information Fig. S5). The prime denotes a derivative with respect to $x$. The angular frequency $\omega_{0}=\sqrt{k / m}$ of the mechanical mode is considered because the frequency shift due to the electromechanical coupling is small $\delta \omega / \omega_{0}=\left(\partial \mathscr{F}_{e} / \partial x\right) / 2 k=0.02 \%$.

The instability threshold relation 3 is used to determine an experimental value for $R^{\prime}$ which can be considered as a piezoresistive coefficient associated to the mechanical vibration. The experimental values are $R_{t}=1 \mathrm{~T} \Omega, \omega_{0}=2 \pi \times 38500 \mathrm{rad} . \mathrm{s}^{-1}, Q=5000$, the later two being measured with forced oscillations without contact and $V_{D C}=0 \mathrm{~V}$. The effective mass $m=5 \times 10^{-15} \mathrm{~kg}$ is calculated from the long resonator density, length and radius. The non-contacted configuration is also used to measure ${ }^{28}$ typical values for the long nanowire capacitance $(C=1 \mathrm{fF})$ and its first derivative $\left(C^{\prime}=1 \mathrm{pF} \cdot \mathrm{m}^{-1}\right)$. For an experimental threshold voltage $V_{t h}=20 \mathrm{~V}$, the self-oscillation condition gives $R^{\prime}=-1.2 \times 10^{16} \Omega \cdot \mathrm{m}^{-1}$. This value is in good agreement with the electrical measurement from Fig. 1 (c) where the order of magnitude for the resistance variation due to the mechanical vibration corresponds to $\Delta R_{D C} / \Delta x=-2.4 \times 10^{16} \Omega \cdot \mathrm{m}^{-1}$. This value is calculated con- 
sidering that the self-oscillation threshold is associated with a current jump $\Delta I$ giving a resistance variation of $\Delta R_{D C}=-V_{D C} \Delta I / I_{D C}{ }^{2}=-1.2 \times 10^{11} \Omega$ for a typical vibration amplitude $\Delta x=5 \mu \mathrm{m}$.

In-depth studies outside the scope of this article would be necessary to understand the resistance variation at a microscopic level. There are two difficulties in comparing with published studies on point-contacts. Firstly our surfaces are not characterized. Secondly, and more specifically, here the contact is varied by torques between the nanowires while studies that combine force and electrical measurements are generally for vertical forces between surfaces. Nevertheless under certain assumptions one can make order of magnitude comparisons with point-contact studies between metals. These are known to show nonlinear current-voltage and current-position characteristics. At the contact position, the typical current variation ${ }^{30}$ for a small change in surface-surface distance $\delta \sim 0.1 \AA$ is $\Delta I \sim 1 \mathrm{nA}$ which gives $\Delta R / \delta \sim 3 \times 10^{17} \Omega . \mathrm{m}^{-1}$. For the contacted nanowires, the mechanical oscillation $x$ is likely to modulate the nanowire-nanowire distance $\delta$ at the point-contact (see supporting information, inserted view in Fig. S6). For simplicity, the scaling law between $\delta$ and $x$ is considered and corresponds geometrically to the ratio between the nanowires typical diameter $d$ and the free length for the long nanowire $\delta=\left(d /\left[(1-79 \%) L_{l}\right]\right) x$. With $d=200 \mathrm{~nm}$, $\delta=4.8 \times 10^{-3} x$ one finds $\Delta R / x=1.4 \times 10^{15} \Omega . \mathrm{m}^{-1}$ which is compatible with the piezoresistance value $R^{\prime}$ obtained before.

The self-oscillation is observed only when a vibration node of the long resonator is close to or at the position of the contact because then the short nanowire does not need to move, which would add strong supplementary rigidity and block the vibrations. As well, the short nanowire vibration would involve a supplementary damping partly due to ohmic losses through the large resistance of the short nanowire ${ }^{28}$. The node at the contact also insures a maximal pivoting movement of the point-contact while a collective displacement of the nanowires would have no effect on the point-contact deformation.

The necessity to contact at a vibration node might appear to be a serious experimental difficulty. An important property of our method is the possibility to adjust the vibration node position with an induced mechanical longitudinal tension $T$ due to the displacement in the $\mathrm{X}$ direction of 
the short nanowire. This is particularly interesting when the contact is not precisely at $79 \%$ of the long resonator length corresponding to the vibration node without mechanical tension $(T=0)$. A modeling of the mechanical coupling for the contacted nanowires is used to define the contact positions and mechanical tensions for which a vibration node is at the contact position (see supporting information). The results are shown for the second mode (solid line) and the third mode (dashed line) in Fig. 3. The node of the second mode can be made to correspond to any contact position from $78 \%$ to $100 \%$ of the long resonator length, providing that the appropriate mechanical tension is applied. The experimental data points correspond to self-oscillation observations using an image analysis for the determination of the contacting position and the mechanical tension value. The mechanical tension $T$ induced in the long resonator is calculated from the static deflection $\delta_{r}$ and the spring coefficient $k$ of the short nanowire.

The lowest threshold voltage was measured in the buckling region discussed in Fig. 3 and its caption to be $V_{D C}=2 \mathrm{~V}$ with $I_{D C}=0.5 \mathrm{pA}$, meaning that the input power of the DC source was $P_{\text {in }}=1 \mathrm{pW}$. This value is remarkably low, particularly for a first demonstration of the phenomena and is the lowest reported value leading to a self-oscillating NEMS (see Fig. 4, horizontal axis). As expected, the input power is larger for self-oscillating NEMS with macroscopic external coupling such as amplifiers (Feng, Villanueva, Ramos) or optical (Metzger, Barton) and microwave (Teufel) cavities for which the input power is typically around $1 \mu \mathrm{W}$ to $1 \mathrm{~mW}$. For nanoscale feedback self-oscillators, solid state devices also require large input power because of large currents drawn through their low resistances (Grogg, Steeneken) while for optical carrier excitation appreciable laser beam power is used (Okamoto). The field-emitting self-oscillators (Ayari, Weldon) have much lower current but suffer from large extraction voltages. At first sight a low input power can be expected for ideal charge shuttle self-oscillators because just below the threshold there are no current and no dissipative losses. However the only experimentally operating device (Kim) showed additional losses due to parasitic field-emission.

The input power is compared to the mechanical power losses as a more relevant way to rate the efficiency of the different self-oscillation mechanisms (Fig. 4, vertical axis). In general any 
self-oscillation mechanism acting on a passive resonator can be seen as an active compensation of the intrinsic mechanical losses. Considering these mechanical losses as inevitable, the efficiency $\eta$ of the self-oscillation mechanism is introduced as the ratio between the intrinsic mechanical power losses during a cycle and the input power, $\eta=P_{d i s s} / P_{i n}$. For an optimal self-oscillation mechanism $(\eta=1)$, the input power exactly equals the mechanical losses meaning that the minimal power is injected to overcome the intrinsic dissipation. During self-oscillation for a quasi-harmonic vibration, the intrinsic mechanical power losses are given by $P_{d i s s}=m \omega_{0} / Q<\dot{x}>_{r m s}{ }^{2}$, where $\langle\dot{x}\rangle_{r m s}$ is the root mean square velocity for vibration cycle with typical amplitude $A, m$ the effective mass, $\Gamma=\omega_{0} / Q$ the intrinsic damping. For the contacted nanowires, the self-oscillation frequency is measured with the position detection technique $(\omega / 2 \pi=17.4 \mathrm{kHz})$. This value is smaller than the non contacted value $(38.5 \mathrm{kHz})$ because the buckling instability lowers the stiffness of the resonator. The quality factor $(Q=5000)$ is measured without a DC voltage to avoid the self-oscillation regime. For an amplitude $A \sim 3 \mu \mathrm{m}$, the power losses are found to be $P_{\text {diss }}=$ $m \omega_{0} / Q \omega^{2} A^{2} / 2=13 \mathrm{fW}$ which means that the efficiency of our self-oscillation mechanism is $\eta=1.3 \%$. Though modest, this value is larger than the estimated efficiencies of any other reported self-oscillating NEMS devices (Fig. 4). Our self-oscillator shows the lowest input power and the best efficiency because of the large resistances that prevent dissipative currents and also because the point-contacts have large piezoresistivity which is the origin of the self-oscillation mechanism.

In conclusion, two nanowires simply brought into contact have been used to demonstrate the existence of a new self-oscillation mechanism for NEMS devices. The self-oscillation is caused by an electromechanical coupling under bias voltage where the large piezoresistance $R^{\prime}$ at the contact is the origin of a negative damping term that overcomes the natural damping at the threshold voltage. By varying the position of the contact point and the static mechanical stress, the selfoscillation was observed for an input power as small as $1 \mathrm{pW}$ which is much smaller than the power consumption of any other self-oscillation mechanism in NEMS devices. Furthermore, the self-oscillation regime exists for relatively low applied voltages, i.e. typically 10 Volts, not far from CMOS. It should also be noted that the derived devices can have very small footprints because the 
elements are all at the nanoscale and the frequency can be selected over a wide range by simply choosing the nanowire dimensions. The easy fabrication, low power consumption, low operating voltages, small footprint and frequency variability together mean that these self oscillating NEMS are serious candidates for integration into portable devices.

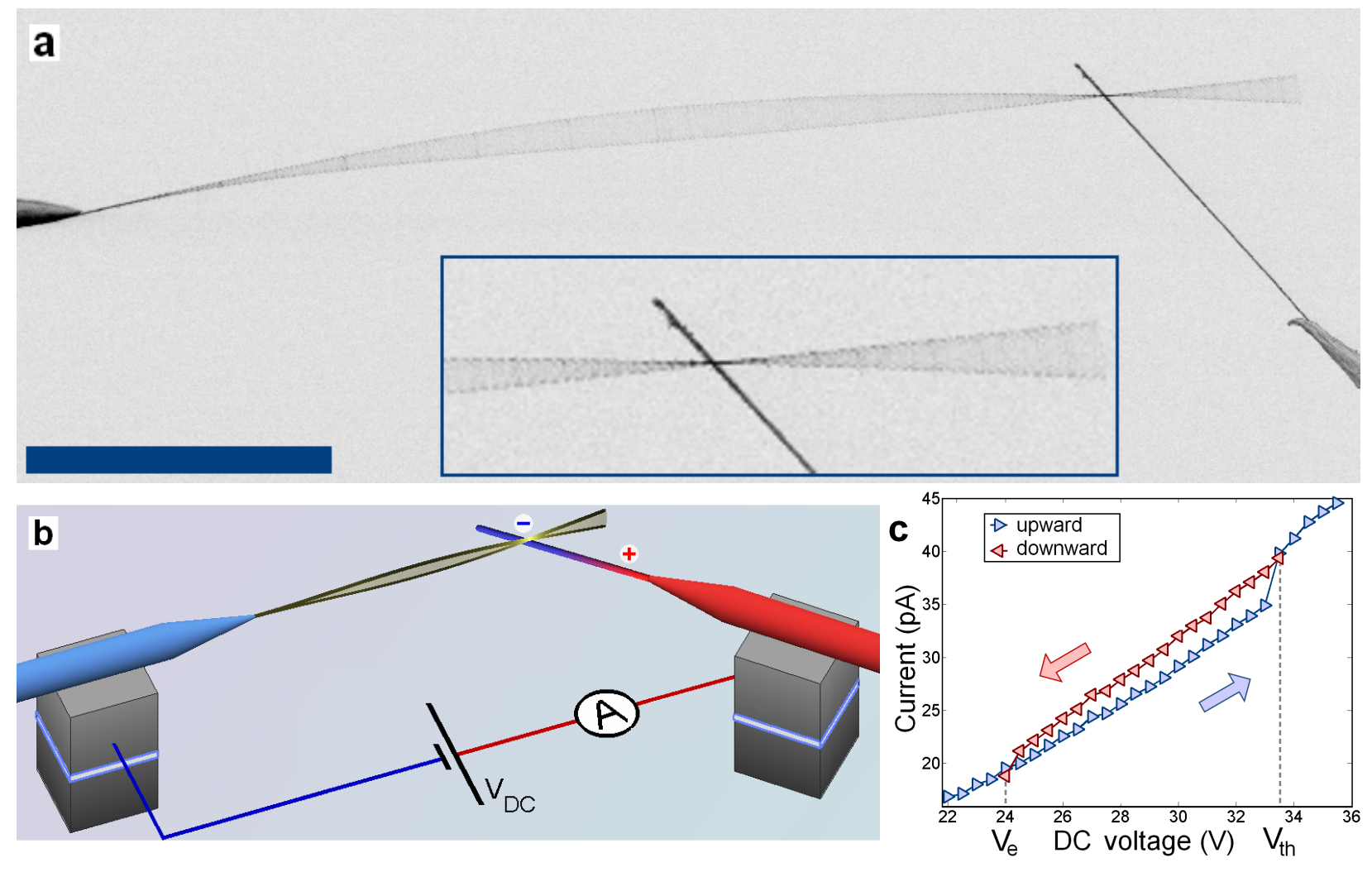

Figure 1: (a) SEM observation of a spontaneous mechanical oscillation of contacted SiC nanowires. The image pixels are numerically inverted which means that the dark regions correspond to a larger collection of secondary electrons. The inserted image is a $3 \times$ zoom of the contacted region. $V_{D C}=27 \mathrm{~V}, I_{D C}=30 \mathrm{pA}$. Scale bar $50 \mu \mathrm{m}$. (b) Schematic representation of the experimental setup. A DC voltage is applied between the supports of the nanowires and generates the self-oscillation of the long resonator. The DC current is measured with a series ammeter. (c) DC current-voltage measurement during a self-oscillation hysteresis cycle $\left(V_{e}=24 \mathrm{~V}, V_{t h}=33,5 \mathrm{~V}\right)$. 


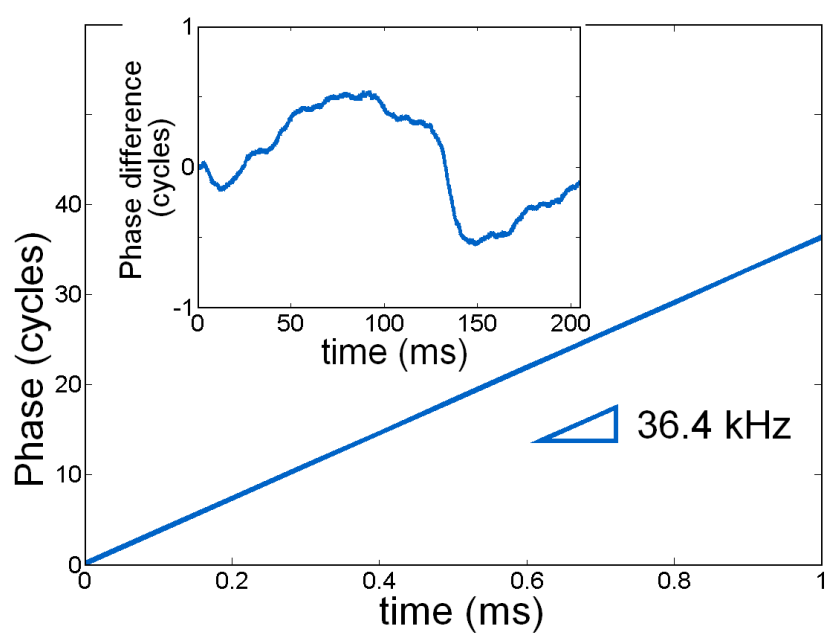

Figure 2: Time evolution of the self-oscillator number of cycles $(\Pi(t)$ in the text) recorded with the focused beam detection technique. The linear slope is the average self-oscillation frequency $(<\mathrm{f}\rangle$ $=36.4 \mathrm{kHz}$ ). (insert) Evolution of the relative number of cycles with time $(\Delta \Pi(t))$. This plot is obtained from the main plot after subtraction of the linear time dependence $(\Delta \Pi(t)=\Pi(t)-<f>$ $t)$. The acquisition time $(200 \mathrm{~ms})$ for the data in the inserted plot corresponds to 7500 oscillation cycles where the relative number of cycles fluctuates with a typical amplitude of one cycle. 


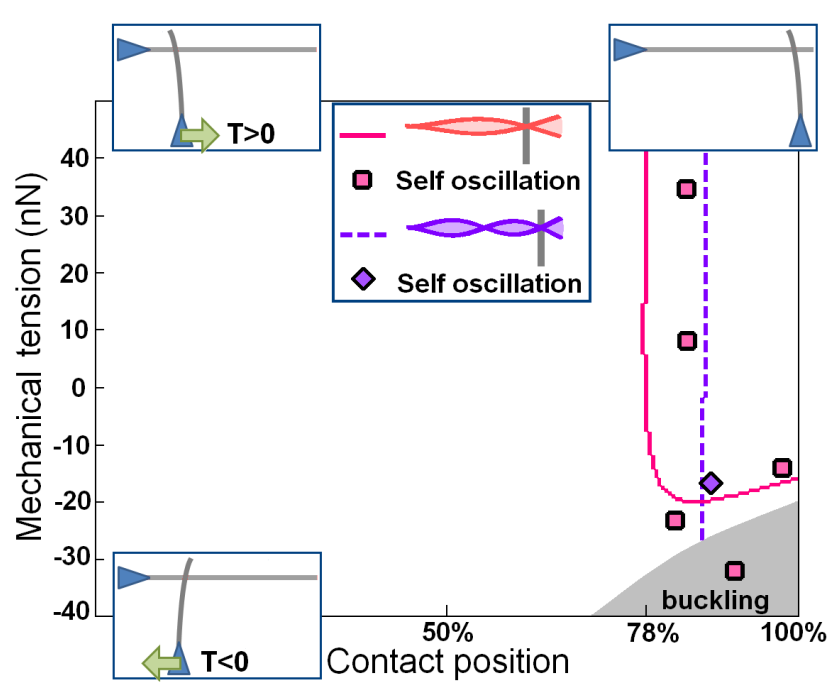

Figure 3: 2D map where the $\mathrm{x}$-axis is the position of the contact on the long resonator (as a ratio of the length) and the $y$-axis is the mechanical longitudinal tension $T$ induced in the long resonator by the lateral displacement of the short nanowire. When the contact is made near the end of the long resonator, a strong negative tension leads to a buckling instability (grey area). The continuous and dashed lines are numerical computations corresponding to contacting positions and mechanical tensions for which a mechanical vibration node matched the contacting position. The experimental data points represent 6 observed self-oscillating states. For each point, the contact position on the long resonator and the deflection of the short nanowire $\delta_{r}$ are determined by image analysis. The mechanical tension is taken from the relation $T=k \delta_{r}$, where $k=6.3 \mathrm{mN} \cdot \mathrm{m}^{-1}$ is the spring coefficient for the short nanowire deflection.

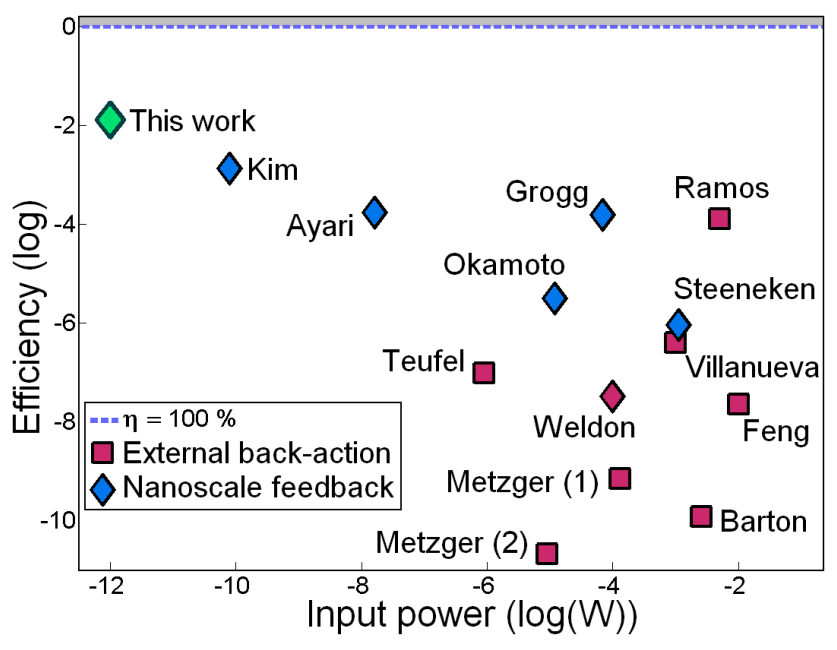

Figure 4: Efficiency of self-oscillation mechanisms, $\eta=P_{\text {diss }} / P_{i n}$, as a function of the input power $P_{i n} . P_{d i s s}$ is the power dissipation by the intrinsic mechanical losses. The values for the expression of $P_{\text {diss }}$ and $P_{\text {in }}$ are displayed in table 1. The dashed line corresponds to an efficiency 1 ( 0 in logarithmic axis) for which all the driving power would compensate the mechanical losses. 
Table 1: Power dissipation by the intrinsic mechanical losses $\left(P_{\text {diss }}\right)$ and input power $\left(P_{\text {in }}\right)$ for reported MEMS/NEMS self-oscillators. The power dissipated by the mechanical motion is calculated for each device using $P_{\text {diss }}=\Gamma<\dot{x}>_{r m s}{ }^{2}$, with the damping coefficient $\Gamma=2 \pi m f / Q$ and the RMS velocity for a quasi harmonic oscillation $\left\langle\dot{x}>_{r m s}=2 \pi f A / \sqrt{2}\right.$ ( $f$ : oscillator frequency, $Q$ : quality factor, $m$ : effective mass, $A$ : typical amplitude during self-oscillation).

\begin{tabular}{|r|c|r|r|r|r|l|}
\hline$f$ & $Q$ & $m(\mathrm{~kg})$ & $A$ & $P_{\text {in }}$ & $P_{\text {diss }}$ & Ref. \\
\hline $17.4 \mathrm{kHz}$ & 5000 & $5 \times 10^{-15}$ & $3 \mu \mathrm{m}$ & $1 \mathrm{pW}$ & $23 \mathrm{fW}$ & This work \\
\hline $428 \mathrm{MHz}$ & 2500 & $5.8 \times 10^{-17}$ & $1 \mathrm{~nm}$ & $10 \mathrm{~mW}$ & $225 \mathrm{pW}$ & Feng $^{10}$ \\
$14.3 \mathrm{MHz}$ & 1220 & $1.3 \times 10^{-14}$ & $10 \mathrm{~nm}$ & $1 \mathrm{~mW}$ & $396 \mathrm{pW}$ & Villanueva $^{11}$ \\
$1.5 \mathrm{MHz}$ & 300000 & $6.2 \times 10^{-15}$ & $100 \mathrm{~nm}$ & $900 \mathrm{nW}$ & $87 \mathrm{fW}$ & Teufel $^{12}$ \\
$7.3 \mathrm{kHz}$ & 2000 & $3.8 \times 10^{-12}$ & $1 \mu \mathrm{m}$ & $130 \mu \mathrm{W}$ & $92 \mathrm{fW}$ & Metzger $(1)^{13}$ \\
$8.7 \mathrm{kHz}$ & 1800 & $3.3 \times 10^{-12}$ & $35 \mathrm{~nm}$ & $9 \mu \mathrm{W}$ & $0.18 \mathrm{fW}$ & ${\text { Metzger }(2)^{15}}^{15}$ \\
$5 \mathrm{MHz}$ & 500 & $1 \times 10^{-16}$ & $10 \mathrm{~nm}$ & $2.6 \mathrm{~mW}$ & $310 \mathrm{fW}$ & Barton $^{16}$ \\
$39.4 \mathrm{kHz}$ & 2.5 & $5.3 \times 10^{-11}$ & $2 \mu \mathrm{m}$ & $5 \mathrm{~mW}$ & $640 \mathrm{nW}$ & Ramos $^{17}$ \\
\hline $42 \mathrm{kHz}$ & 4000 & $1.2 \times 10^{-14}$ & $10 \mu \mathrm{m}$ & $17 \mathrm{nW}$ & $2.8 \mathrm{pW}$ & Ayari $^{18}$ \\
$4 \mathrm{MHz}$ & 1260 & $5 \times 10^{-19}$ & $1 \mu \mathrm{m}$ & $100 \mu \mathrm{W}$ & $3.2 \mathrm{pW}$ & Weldon $^{20}$ \\
$10.5 \mathrm{MHz}$ & 500 & $4.1 \times 10^{-19}$ & $30 \mathrm{~nm}$ & $80 \mathrm{fW}$ & $110 \mathrm{fW}$ & Kim $^{22}$ \\
$3.6 \mathrm{MHz}$ & 600 & $3.1 \times 10^{-13}$ & $60 \mathrm{~nm}$ & $70 \mu \mathrm{W}$ & $11 \mathrm{nW}$ & Grogg $^{23}$ \\
$1.26 \mathrm{MHz}$ & 10000 & $4.1 \times 10^{-12}$ & $100 \mathrm{~nm}$ & $1.1 \mathrm{~mW}$ & $1 \mathrm{nW}$ & Steeneken $^{24}$ \\
$239 \mathrm{kHz}$ & 6500 & $3.5 \times 10^{-12}$ & $2 \mu \mathrm{m}$ & $12 \mu \mathrm{W}$ & $37 \mathrm{pW}$ & Okamoto $^{25}$ \\
\hline
\end{tabular}




\section{References}

(1) O’Connell, A. D.; Hofheinz, M.; Ansmann, M.; Bialczak, R. C.; Lenander, M.; Lucero, E.; Neeley, M.; Sank, D.; Wang, H.; Weides, M.; Wenner, J.; Martinis, J. M.; Cleland, A. N. Nature 2010, 464, 697.

(2) Teufel, J. D.; Donner, T.; Li, D.; Harlow, J. W.; Allman, M. S.; Cicak, K.; Sirois, A. J.; Whittaker, J. D.; Lehnert, K. W.; Simmonds, R. W. Nature 2011, 475, 359.

(3) Chan, J.; Mayer Alegre, T. P.; Safavi-Naeini, A. H.; Hill, J. T.; Krause, A.; Gröblacher, S.; Aspelmeyer, M.; Painter, O. Nature 2011, 478, 89.

(4) Steele, G. A.; Hüttel, A. K.; Witkamp, B.; Poot, M.; Meerwaldt, H. B.; Kouwenhoven, L. P.; van der Zant, H. S. J. Science 2009, 325, 1103.

(5) Lassagne, B.; Tarakanov, Y.; Kinaret, J.; Garcia-Sanchez, D.; Bachtold, A. Science 2009, $325,1107$.

(6) Chaste, J.; Eichler, A.; Moser, J.; Ceballos, G.; Rurali, R.; Bachtold, A. Nature Nanotechnology 2012, 7, 301.

(7) Jensen, K.; Weldon, J.; Garcia, H.; Zettl, A. Nano Lett. 2007, 7, 3508.

(8) Rutherglen, C.; Burke, P. Nano Lett. 2007, 7, 3296.

(9) Gouttenoire, V.; Barois, T.; Perisanu, S.; Leclercq, J.-L.; Purcell, S. T.; Vincent, P.; Ayari, A. Small 2010, 6, 1060.

(10) Feng, X. L.; White, C. J.; Hajimiri, A.; Roukes, M. L. Nature 2008, 3, 342.

(11) Villanueva, L. G.; Karabalin, R. B.; Matheny, M. H.; Kenig, E.; Cross, M. C.; Roukes, M. L. Nano Lett. 2011, 11, 5054.

(12) Teufel, J. D.; Harlow, J. W.; Regal, C. A.; Lehnert, K. W. Phys. Rev. Lett. , 2008, 101, 197203. 
(13) Metzger, C. H.; Karrai, K. Nature 2004, 432, 1002.

(14) Arcizet, O.; Cohadon, P.-F.; Briant, T.; Pinard, M.; Heidmann, A. Nature 2006, 444, 71.

(15) Metzger, C.; Ludwig, M.; Neuenhahn, C.; Ortlieb, A.; Favero, I.; Karrai, K.; Marquardt, F. Phys. Rev. Lett. 2008, 101, 133903.

(16) Barton, R. A.; Storch, I. R.; Adiga, V. P.; Sakakibara, R.; Cipriany, B. R.; Ilic, B.; Wang, S. P.; Ong, P.; McEuen, P. L.; Parpia, J. M.; Craighead, H. G. Nano Lett. 2012, 12, 4681.

(17) Ramos, D.; Mertens, J.; Calleja, M.; Tamayo, J. Appl. Phys. Lett. 2008, 92, 173108.

(18) Ayari, A.; Vincent, P.; Perisanu, S.; Choueib, M.; Gouttenoire, V.; Bechelany, M.; Cornu, D.; Purcell, S. T. Nano Lett. 2007, 7, 2252.

(19) Kleshch, V. I.; Obraztsov, A. N.; Obraztsova, E. D. Carbon 2010, 48, 3895.

(20) Weldon, J. A.; Alemán, B.; Sussman, A.; Gannett, W.; Zettl, A. K. Nano Lett. 2010, 10, 1728.

(21) Park, H.; Park, J.; Lim, A. K. L.; Anderson, E. H.; Alivisatos, A. P.; McEuen, P. L. Nature 2000, 407, 57.

(22) Kim, H. S.; Qin, H.; Blick, R. H. New Journal of Physics 2010, 12, 033008.

(23) Grogg, D.; Ayöz, S.; Ionescu, A. M. Self-sustained Low Power Oscillator Based on Vibrating Body Field Effect Transistor. 2009.

(24) Steeneken, P. G.; Phan, K. L.; Goossens, M. J.; Koops, G. E. J.; Brom, G. J. A. M.; van der Avoort, C.; van Beek, J. T. M. Nature Phys. 2011, 7, 354.

(25) Okamoto, H.; Ito, D.; Onomitsu, K.; Sanada, H.; Gotoh, H.; Sogawa, T.; Yamaguchi, H. Phys. Rev. Lett. 2011, 106, 036801.

(26) Loh, O. Y.; Espinosa, H. D. Nature Nanotechnology 2012, 7, 283.

(27) Jansen, A. G. M.; Gelder (van), A. P.; P, W. J. Phys. C 1980, 13, 6073. 
(28) Barois, T.; Ayari, A.; Siria, A.; Perisanu, S.; Vincent, P.; Poncharal, P.; Purcell, S. T. Phys. Rev. B 2012, 85, 075407.

(29) Vincent, P.; Perisanu, S.; Ayari, A.; Choueib, M.; Gouttenoire, V.; Bechelany, M.; Brioude, A.; Cornu, D.; Purcell, S. T. Phys. Rev. B 2007, 76, 085435.

(30) Gimzewski, J. K.; Möller, R. Phys. Rev. B 1987, 36, 1284. 